\title{
Accelerated 4D flow imaging with variable- density cartesian undersampling and parallel imaging reconstruction
}

\author{
Jing Liu ${ }^{{ }^{*}}$, Petter Dyverfeldt ${ }^{2}$, Michael D Hope ${ }^{1}$, David Saloner ${ }^{1}$ \\ From 16th Annual SCMR Scientific Sessions \\ San Francisco, CA, USA. 31 January - 3 February 2013
}

\section{Background}

4D flow CMR is hampered by long scan times. We investigated an effective undersampling scheme and an efficient parallel image reconstruction method to achieve highly accelerated 4D flow CMR with high reconstruction accuracy.

\section{Methods}

Variable-density Poisson Disk Distribution (VD-PDD) undersampling was applied for 4D flow imaging. By applying VD-PDD independently at each time frame (Figure 1a), we achieved random undersampling in both the ky-kz plane and temporal domains. In addition, we applied an improved initial solution for SPIRIT to significantly improve reconstruction accuracy and robustness.

We explored the different undersampling and reconstruction algorithms on fully sampled 4D flow CMR data acquired on a $1.5 \mathrm{~T}$ Siemens Avanto scanner with a 5-ch coil in 3 volunteers $($ venc $=200 \mathrm{~cm} / \mathrm{s}, \mathrm{FOV}=320 \times 240 \times 55$ $\mathrm{mm}^{3}$, matrix $=128 \times 96 \times 22, \sim 18$ time frames of $35 \mathrm{~ms}$ temporal resolution, $\sim 25$ mins scan time). VD-PDD (center $12 \times 12$ fully sampled; $R=6$ ) was retrospectively applied to the full data.

Composite data was generated by sharing data from other frames based on the temporal distance to the time frame of interest (Figure 1b). k-space was filled to the extent possible from selected adjacent time frames. The images reconstructed from the composite data through time (Figure 1c) were used as initial solutions for SPIRIT. This is denoted as method "M1". We also generated composite data that shared limited data from other frames (Figure 1d) to mitigate undersampling of the data. Then

${ }^{1}$ Radiology and Biomedical Imaging, University of California San Francisco, San Francisco, CA, USA

Full list of author information is available at the end of the article
SPIRIT with the proposed initial solutions was applied to this new composite data through time (Figure 1e), referred to as "M2". Flow-waveforms in the ascending (AA) and descending aorta (DA) were measured in 5 locations in each subject (Figure 2d-e). Relative error was calculated with the fully sampled data as reference.

\section{Results}

Both reconstruction methods were successfully applied to all subjects. Velocity images compared favorably to the fully sampled velocity image (Figure 2a-c). The relative error in flow measurement was $0.05 \pm 0.01$ (AA, M1), 0.04 \pm 0.01 (AA, M2), $0.09 \pm 0.05$ (DA, M1), and $0.07 \pm 0.02$ (DA, M2). Both proposed methods achieved outstanding performance with 6-fold acceleration. Of the two, M2 had smaller errors, indicating that an effective temporal sharing scheme combined with VD-PDD and SPIRIT could be a potential way of improving image quality without sacrificing temporal resolution.

\section{Conclusions}

We employed undersampling patterns based on VD-PDD, parallel imaging method SPIRIT, and a temporal sharing scheme to achieve 6-fold accelerated 4D flow CMR with a small number of coils. The qualitative and quantitative comparisons indicate the potential of our methods to achieve highly accelerated flow imaging with maintained accuracy. Future work includes implementation of prospective undersampling.

\section{Funding}

American Heart Association BGIA.

NIH NIBIB K25. 


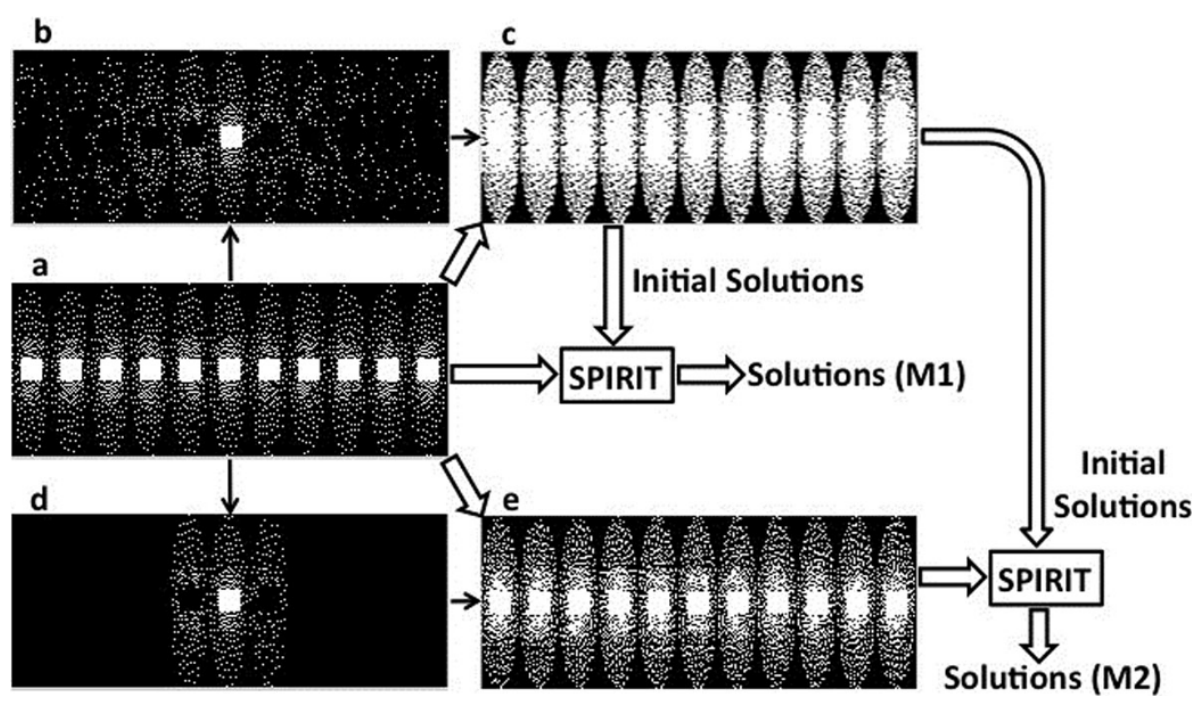

Figure 1 a) Variable-density Poisson-Disk Distribution sampling on ky-kz plane through time (each block is for one time frame; k-space center is fully sampled), b) the selected samples at each time frame for generating a composite data used for the middle frame, c) composite sampling patterns through time for generating initial solutions for SPIRIT, d) the selected samples for generating a composite image for the middle frame, e) new composite data with reduced undersampling.

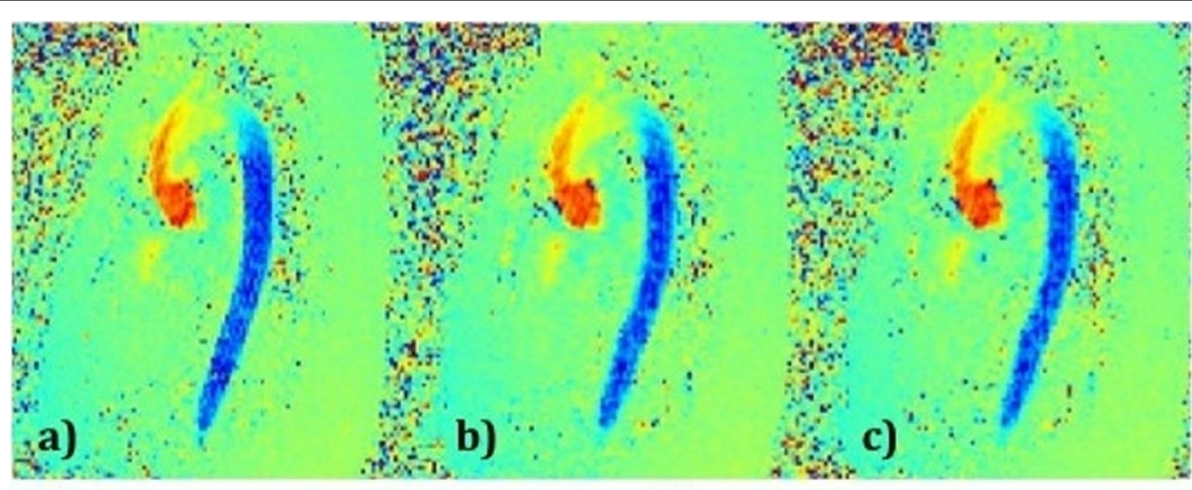

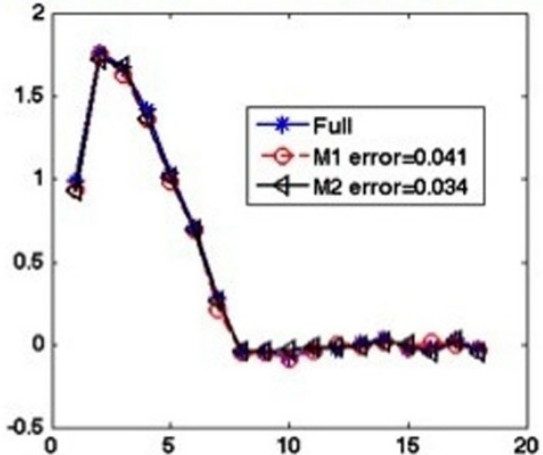

d)

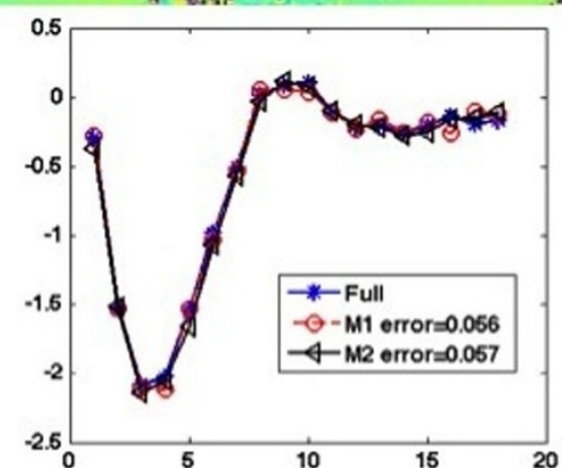

e)

Figure 2 Flow images obtained with a) $R=1$ (full), b) $R=6$ VD-PDD and SPIRIT with improved initial solution (M1), and c) R=6 VD-PDD with reduced undersampling and SPIRIT with improved initial solution (M2). d-e) show representative flow-waveforms of AA and DA with full data, $\mathrm{M} 1$ and $\mathrm{M} 2$. 


\section{Author details}

${ }^{1}$ Radiology and Biomedical Imaging, University of California San Francisco,

San Francisco, CA, USA. ${ }^{2}$ Linköping University Hospital, Linköping, Sweden.

Published: 30 January 2013

\section{References}

1. Lustig M, et al:. MRM 2010, 64:457-71.

2. Murphy MJ, et al.: IEEE BMI 2011, 1039-43.

doi:10.1186/1532-429X-15-S1-P11

Cite this article as: Liu et al:: Accelerated 4D flow imaging with variabledensity cartesian undersampling and parallel imaging reconstruction.

Journal of Cardiovascular Magnetic Resonance 2013 15(Suppl 1):P11.

Submit your next manuscript to BioMed Central and take full advantage of:

- Convenient online submission

- Thorough peer review

- No space constraints or color figure charges

- Immediate publication on acceptance

- Inclusion in PubMed, CAS, Scopus and Google Scholar

- Research which is freely available for redistribution

Submit your manuscript at www.biomedcentral.com/submit
() Biomed Central 